\title{
Effects of Dexmedetomidine on the
}

\section{Pharmacokinetics of Dezocine, Midazolam and Its Metabolite I-Hydroxymidazolam in Beagles by UPLC-MS/MS}

This article was published in the following Dove Press journal:

Drug Design, Development and Therapy

\author{
Wei Zhou ${ }^{1,2}$ \\ Shuang-long $\mathrm{Li} \mathbb{D}^{3}$ \\ Ti Zhao ${ }^{3}$ \\ Le $\mathrm{Li}^{3}$ \\ Wen-bin Xing ${ }^{3}$ \\ Xiang-jun $\mathrm{Qiu}^{3}$ \\ Wei Zhang' \\ 'The First Affiliated Hospital of \\ Zhengzhou University, Zhengzhou \\ 450052, People's Republic of China; \\ ${ }^{2}$ Nanyang City Central Hospital, Nanyang \\ 473009, People's Republic of China; \\ ${ }^{3}$ School of Basic Medicine, Henan \\ University of Science and Technology, \\ Luoyang 47I023, People's Republic of \\ China
}

Objective: We developed and validated a sensitive and reliable UPLC-MS/MS method for simultaneous determination of dezocine (DEZ), midazolam (MDZ) and its metabolite 1hydroxymidazolam (1-OH-MDZ) in beagle plasma and investigated the effect of dexmedetomidine (DEX) on the pharmacokinetics of DEZ, MDZ and 1-OH-MDZ in beagles.

Materials and Methods: Diazepam was used as the internal standard (IS); the three analytes and IS were extracted by acetonitrile precipitation and separated on an Acquity UPLC BEH C18 column using acetonitrile- $0.1 \%$ formic acid as mobile phase in gradient mode. In positive ion mode, the three analytes and IS were monitored by multiple reaction monitoring (MRM). Six beagles were designed as a double cycle self-control experiment with $0.15 \mathrm{mg} / \mathrm{kg}$ in the first cycle (Group A). After a 1-week washout period, the same six beagles were slowly injected intravenously with $2 \mu \mathrm{g} / \mathrm{kg}$ DEX in the second cycle (Group B), with continuous injection for 7 days. On the seventh day, $0.5 \mathrm{hr}$ after intravenous injection of $2 \mu \mathrm{g} / \mathrm{kg} \mathrm{DEX}$, the six beagles were intramuscularly given with DEZ $0.33 \mathrm{mg} / \mathrm{kg}$ and MDZ $0.15 \mathrm{mg} / \mathrm{kg}$.

Results: Under the conditions of this experiment, this method exhibited a good linearity for each analyte. The accuracy and precision were all within the acceptable limits in the bioanalytical method, and the results of recovery, matrix effect and stability have also met the requirements.

Conclusion: The developed UPLC-MS/MS method for simultaneous determination of DEZ, MDZ and 1-OH-MDZ in beagles plasma was accurate, reproducible, specific, and suitable. DEX could inhibit the metabolism of DEZ and MDZ and increase the exposure of DEZ and MDZ in beagles. Therefore, the change of therapeutic effect and the occurrence of adverse reactions caused by drug-drug interaction should be paid attention to when the drugs were used in combination.

Keywords: dexmedetomidine, dezocine, midazolam, 1-hydroxymidazolam, pharmacokinetics, drug-drug interactions

\section{Introduction}

Drug-drug interactions (DDIs) often occur, especially for patients with multiple underlying diseases who use various kinds of drugs. In clinical practice, polypharmacy is a common problem and results in the increased risks of DDIs. ${ }^{1}$ Ultimately, taking multiple medications simultaneously increases an individual's risk for undesirable DDIs that lead to serious and debilitating adverse drug reactions (ADRs). ${ }^{2} \mathrm{~A}$

\footnotetext{
Xiang-jun Qiu

School of Basic Medicine, Henan

University of Science and Technology,

Luoyang 47I023, People's Republic of

China

Email lyxiangjun@126.com (1)
}

Correspondence: Wei Zhang

Corst Affiliated Hospital of Zhengzhou 
primary reason of DDIs is the change of the cytochrome P450 (CYP450) isozyme activity by inducing or inhibiting. In pharmacokinetics (PK) interactions, one drug affects the absorption, distribution, metabolism, or excretion of another concomitant drug. DDIs through PK occur through drug-metabolizing enzymes and drug transporters that are found within these cells. ${ }^{3}$

Dexmedetomidine (DEX) is a highly selective $\alpha 2$-adrenoceptor agonist with sedative, anxiolytic, sympatholytic and analgosedative properties. $^{4}$ Dexmedetomidine is mainly administered by continuous intravenous infusion at clinical treatment, which needed to be monitored in non- intubated patients owing to the risk of respiratory depression and in some case apnea. ${ }^{5}$ Currently, DEX is licensed in Europe and the USA for analgosedation in ventilated adult ICU patients and procedural sedation in non-ventilated adults. ${ }^{6}$ The application of DEX in the perioperative period of cesarean section was not only conducive to the early conversion of infant feeding to exclusive breastfeeding but could also improve the recovery quality and comfort of the parturient, optimize

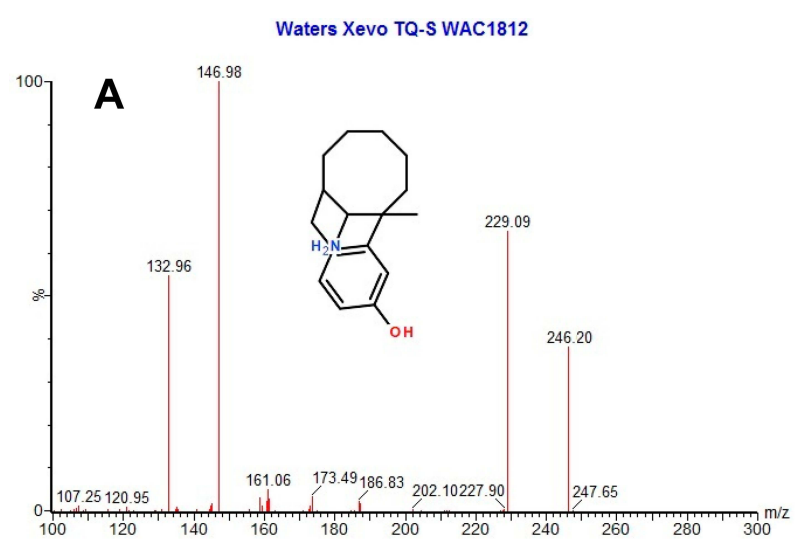

Waters Xevo TQ-S WAC1812

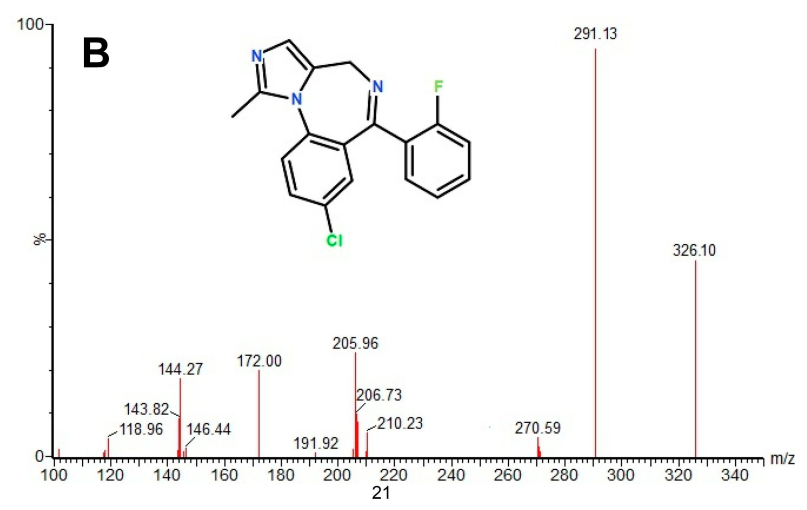

analgesia, shorten the time to first lactation, and increase lactation. ${ }^{7}$ After intravenous administration, DEX quickly distributes beyond total body water. DEX is metabolised by glucuronidation and CYP2A6 hydroxylation and subsequently excreted in urine almost exclusively as metabolite. ${ }^{8,9}$

Dezocine (DEZ, Figure 1A) is a marketed opioid analgesic of the benzomorphan group. It acts as a modulator of $\mu$-, $\delta$-, and $\kappa$-opioid receptors. ${ }^{10} \mathrm{DEZ}$ is a mixed agonist/antagonist of opioid receptors. ${ }^{11}$ It is related to other benzomorphans such as pentazocine, with a similar profile of effects that include analgesia and euphoria. DEZ is absorbed rapidly after intramuscular injection. DEZ can mitigate pain for postoperative patients, prevent propofol injection pain and mitigate its severity, and its efficacy shows no significant difference from that of lidocaine. ${ }^{12}$

Midazolam (MDZ, Figure 1B) is a medication used for anesthesia, procedural sedation, trouble sleeping, and severe agitation. ${ }^{13}$ It belongs to the benzodiazepine class of drugs and works by increasing the activity of the GABA neurotransmitter in the brain. Administration of a single
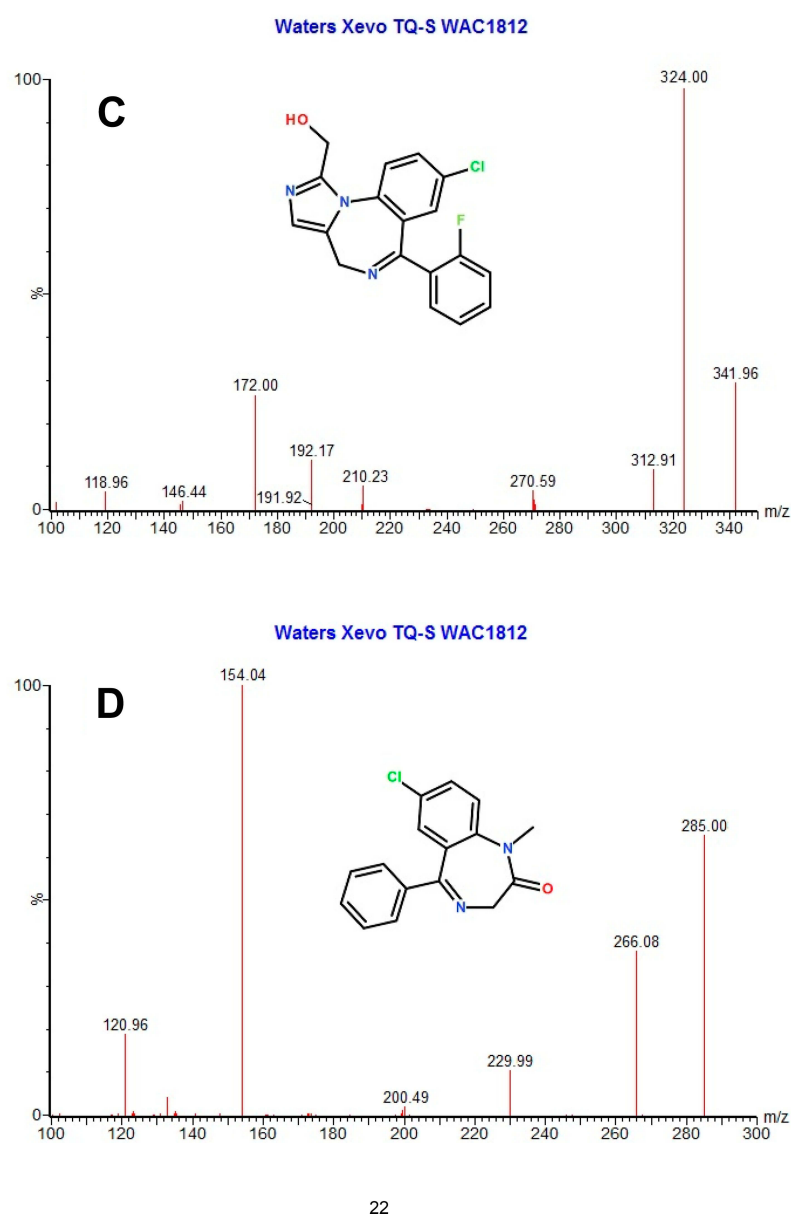

Figure I The chemical structure and the ion transitions from parent ion to daughter ion of three analytes and IS (A- DEZ, B- MDZ, C- I-OH MDZ, D- IS). 
dose of $0.1 \mathrm{mg} / \mathrm{kg} \mathrm{MDZ}$ dose significantly decreases the incidence of severe emergence agitation at the emergence after sevoflurane anesthesia, oral MDZ in a single dose of $0.5 \mathrm{mg} / \mathrm{kg}$ or greater is associated with successful completion of minor pediatric procedures. ${ }^{14,15} \mathrm{MDZ}$ is considered one of the best CYP3A4 probe drugs, since it is extensively metabolized by CYP3A4 to its major metabolite 1-hydroxymidazolam (1-OH-MDZ, Figure 1C) and rapidly excreted into urine as its glucuronide conjugate. ${ }^{16,17}$

DEX, DEZ and MDZ are all anesthesia-assisted drugs, which may be used simultaneously in clinical practice, DEX may enhance the effects of other sedatives and anesthetics when co- administered. DEX is a strong inhibitor of CYP P450 enzyme. ${ }^{18} \mathrm{MDZ}$ is extensively metabolized by CYP3A4, and DEZ is metabolized by liver, therefore DDIs in PK of the three drugs will occur. In the current research, at first, a sensitive UPLC-MS/MS method for simultaneous determination of DEZ, MDZ, and 1-OH MDZ in beagle plasma was developed, diazepam was used as an internal standard (IS, Figure 1D). Then, the effects of DEX on pharmacokinetics of DEZ, MDZ, and 1-OH MDZ in beagles were studied.

\section{Materials and Methods Chemicals and Reagents}

DEZ (purity $>98.0 \%$ ), MDZ (purity > 98.0\%), 1-OH MDZ (purity $>98.0 \%$ ) were purchased from Sigma (St. Louis, MO, USA). Diazepam (purity $>98.0 \%$, IS) were obtained from China Academy of pharmaceutical and biological products.

DEZ Injection (LOT NO: 18,042,731) was purchased from Yangtze River Pharmaceutical Group Co., Ltd. MDZ Injection (LOT NO: 20,180,311) was purchased from Nhwa Pharma Co., Ltd. DEX Hydrochloride Injection (LOT NO: 180222BP) was obtained from Hengrui medicine Co., Ltd.

\section{Instrumentation and Conditions}

The equipment used in this study included: Waters ACQUITY UPLC instrument (Waters Corp., Milford, MA, USA), XEVO TQD triple quadrupole mass spectrometer (Waters Corp., Milford, MA, USA), Ultrapure water equipment (Millipore, Bedford, USA), Electronic analytical balance and vortex instrument, etc.

Three analytes and IS were separated on an Acquity BEH C18 column $(2.1 \mathrm{~mm} \times 50 \mathrm{~mm}, 1.7 \mu \mathrm{m})$ by gradient elution with the mobile phase of $0.1 \%$ formic acid (A) and acetonitrile (B) at the flow rate of $0.4 \mathrm{~mL} / \mathrm{min}$. The gradient program was as follows: $0.00-0.50 \mathrm{~min}, 10 \% \mathrm{~B}$; 0.50-1.00 min, 10-90\% B; 1.00-2.00 min, 90\% B; 2.00$2.10 \mathrm{~min}, 90-10 \% \mathrm{~B} ; 2.10-3.00 \mathrm{~min}, 10 \% \mathrm{~B}$. The column temperature was set at $45^{\circ} \mathrm{C}$ and the autosampler was conditioned at $4^{\circ} \mathrm{C}$. The injection volume of $2 \mu \mathrm{L}$ was applied for analysis.

The XEVO TQ-S triple quadrupole mass spectrometer was used for Mass spectrometric (MS) measurement with an electrospray ionization (ESI) interface in positive ionization mode. Under the multiple reaction monitoring (MRM) conditions, quantification was achieved with transitions of $m / z 246.20 \rightarrow 147.00$ for DEZ, $m / z 326.10 \rightarrow$ 291.13 for $\mathrm{MDZ}, \mathrm{m} / z 341.96 \rightarrow 324.00$ for $1-\mathrm{OH} \mathrm{MDZ}$, and $m / z 285.00 \rightarrow 154.00$ for IS, respectively. All data were acquired in centroid mode by Masslynx V4.1 software (Waters, Milford, MA, USA). The ion transitions from parent ion to daughter ion of three analytes and IS are shown in Figure 1.

\section{Solutions Ready}

$10 \mathrm{mg}$ of DEZ, MDZ and 1-OH MDZ were accurately weighed, respectively, in three different $10 \mathrm{~mL}$ volumetric flask, dissolved in methanol and volume to the scale, respectively. Through gradient dilution of the original solution, various working solutions of calibration curve and quality control (QC) in methanol were obtained. The final concentrations of the calibration curves were covered by several points as follows: 1, 5, 10, 25, 50, 100, 250 and $500 \mathrm{ng} / \mathrm{mL}$ for DEZ and 1-OH MDZ; 0.5, 1, 2.5, 5, 10, 25, 50 and $100 \mathrm{ng} / \mathrm{mL}$ for MDZ. QC samples in plasma were similarly prepared and the concentrations were set $2.5,50$, and $400 \mathrm{ng} / \mathrm{mL}$ for DEZ and 1-OH MDZ; 1, 10, and $80 \mathrm{ng} /$ $\mathrm{mL}$ for MDZ. All solutions were stored in a refrigerator at $4^{\circ} \mathrm{C}$ and were brought to room temperature before analysis. The internal standard (IS) working solution (100 ng/ $\mathrm{mL}$ ) was also prepared by diluting the stock solution of IS with acetonitrile.

\section{Sample Preparation}

Beagle plasma samples stored at -20 were thawed at room temperature. Samples were prepared by protein precipitation. In brief, $10 \mathrm{~L}$ IS working solution was added to $50 \mathrm{~L}$ plasma in a $1.5 \mathrm{~mL}$ Eppendorf tube, and followed by vortexing for $15 \mathrm{~s}$. The mixture was precipitated by the addition of $200 \mu \mathrm{L}$ acetonitrile and then vortexed for 1.0 min. Finally, the plasma was centrifuged at $15,000 \times \mathrm{g}$ for 15 min to obtain the supernatant, and $2 \mu \mathrm{L}$ supernatant was injected for analysis by the UPLC-MS/MS system. 


\section{Method Validation}

The method was validated for specificity, linearity, precision, accuracy, recovery, and stability. In this experiment, the UPLC-MS/MS method was validated according to the United States Food and Drug Administration (FDA) guidelines. ${ }^{19}$ The specificity, linearity, lower limit of quantitation (LLOQ), matrix effect (ME), recovery, accuracy, precision, and stability under various conditions have been verified for this method.

The specificity was assessed by comparing chromatograms of blank beagle plasma samples, blank plasma spiked with DEZ, MDZ, 1-OH MDZ and IS.

The calibration curves were constructed and validated by analyzing spiked calibration samples for 3 days in a row. The linearity of the assay was assessed by analyzing the calibration curves using least-squares linear regression of the peak area ratios of the analytes to the IS versus the nominal concentration of the calibration standard with a weighed factor $\left(1 / \chi^{2}\right)$. The lower limit of quantification (LLOQ) was selected as the lowest concentration used in the calibration curve. The carry-over was performed by adding DEZ (500 $\mathrm{ng} / \mathrm{mL}$ ), MDZ (100 $\mathrm{ng} / \mathrm{mL}$ ) or 1-OH MDZ (500 $\mathrm{ng} / \mathrm{mL})$ to three blank plasmas, and then injecting a blank sample. In the sample, each analyte should be less than $20 \%$ of the LLOQ.

The accuracy and precision were assessed by the determination of QC samples at four concentration levels (LLOQ, low, medium and high concentration) in six replicates. On the same day, the intra-day precision was calculated, and the inter-day precision was calculated by continuous measurement within 3 days. Precision was defined as the relative standard deviation (RSD, \%) and accuracy as a relative error (RE, \%).

The extraction recovery evaluated was calculated by the ratio of the peak area of QC sample to that of the same concentration solution prepared by mobile phase, and the Matrix effects (ME) were calculated by the ratio of the peak area of QC sample to that of the same concentration solution prepared by blank plasma.

Stability studies in bio-samples were also conducted at three QC levels in several different storage conditions: room temperature for $12 \mathrm{~h},-20^{\circ} \mathrm{C}$ for at least 4 weeks, after three freeze-thaw cycles $\left(-20^{\circ} \mathrm{C}\right.$ to $\left.25^{\circ} \mathrm{C}\right)$, and processed samples at $4^{\circ} \mathrm{C}$ in an auto-sampler tray for $12 \mathrm{~h}$.

\section{Animals}

Six beagles (half male, half female, weighing $6 \pm 2 \mathrm{~kg}$ ) were obtained from Laboratory Animal Center of Henan
University of Science and Technology (Henan, China). The beagles were adapted to the new environment for 7 days in laboratory conditions. Necessary approval from the Institutional Animal Ethics Committee was obtained to carry out the experiments, and the experiment was approved according to the Laboratory animals - guidelines for ethical review of welfare (GB/T 35,892-2018).

\section{Study Design}

Before the test, the beagles had free access to water but were fasted for $12 \mathrm{~h}$. Six beagles were designed as a double cycle self-control experiment. Blood samples (1.0 $\mathrm{mL}$ ) were collected from the forelimb cephalic vein or the small saphenous vein of the hind limb and put into heparinized tubes at $0.08,0.17,0.33,0.67,1,1.5,2,3,4,6,9$ and $12 \mathrm{~h}$ after intramuscular injection of DEZ $0.33 \mathrm{mg} / \mathrm{kg}$ and MDZ $0.15 \mathrm{mg} / \mathrm{kg}$ in the first cycle (Group A). Blood samples were centrifuged for $10 \mathrm{~min}$ at $3000 \times \mathrm{g}$ and the plasma was collected and kept frozen at $-20^{\circ} \mathrm{C}$ until analysis, and the samples belonged to group A.

After 1-week washout period, the same six beagles were injected intravenously slowly with $2 \mu \mathrm{g} / \mathrm{kg}$ DEX in the everyday morning in the second cycle (Group B), continuous injection for 7 days. On the seventh day, $0.5 \mathrm{hr}$ after intravenous injection of $2 \mu \mathrm{g} / \mathrm{kg}$ DEX, the six beagles were given by intramuscular injection with DEZ $0.33 \mathrm{mg} / \mathrm{kg}$ and MDZ $0.15 \mathrm{mg} / \mathrm{kg}$. The blood samples $(1.0 \mathrm{~mL})$ were collected from the forelimb cephalic vein or the small saphenous vein of the hind limb and put into heparinized tubes at $0.08,0.17,0.33,0.67,1,1.5,2,3$, 4, 6, 9 and $12 \mathrm{~h}$ after intramuscular injection of DEZ and MDZ. Plasma was also separated and preserved, and the samples belonged to group B.

\section{Plasma Sample Detection}

The above-developed UPLC-MS/MS method was used to simultaneously detect DEZ, MDZ and its metabolite 1-OH MDZ in beagle plasma of group A and group B. Plasma samples were tested according to analytical batches, and each analytical batch included standard curve and QC samples.

\section{Data Analysis}

The pharmacokinetic parameters of DEZ, MDZ and 1-OH MDZ were calculated by the noncompartmental analysis using the DAS 2.0 software. All data were expressed as mean \pm standard deviation (SD). 


\section{Results \\ Specificity}

Under the above experimental conditions, DEZ, MDZ, 1$\mathrm{OH} \mathrm{MDZ}$ and IS were well separated from endogenous substances. Representative chromatograms of a blank plasma sample (A), a blank plasma sample spiked with three analytes and IS (B), and a beagle sample after administration (C) are shown in Figure 2. DEX and plasma endogenous substances did not interfere with the detection of the three analytes and IS. The retention times of DEZ, MDZ, 1-OH MDZ and IS were 1.16, 1.22, 1.22 and 1.44 min, respectively. The total running time for each sample was $3.0 \mathrm{~min}$.

\section{Linearity and Carry-Over}

Regression equation, correlation coefficients $(\mathrm{R})$ and linearity range are shown in Table 1 . They all exhibited good linearity. All standards met the criteria of $<15 \%$ deviation from nominal concentration. The LLOQ for DEZ, MDZ and 1-OH MDZ in beagle plasma with the signal-to-noise over 10 was $1.00,0.50$ and $1.00 \mathrm{ng} / \mathrm{mL}$, respectively. In this study, the carry-over did not affect the determination of DEZ, MDZ and 1-OH MDZ.

\section{Precision and Accuracy}

Table 2 shows the results obtained for the intra-day and inter-day precision and accuracy of DEZ, MDZ and 1-OH MDZ. The precision (\% RSD) for three analytes under investigation did not exceed $9.63 \%$, the accuracy (\% RE) was in the range from $-6.05 \%$ to $7.78 \%$ at any of the concentrations studied and met the requirements of validation.

\section{Recovery and ME}

The recovery and ME results were investigated and shown in Table 3, which were accepted in accordance with the US FDA guidelines for the method validation to differentiate and qualify the analytes in a sample. No ME was observed to influence the determination of each analyte in beagle plasma.

\section{Stability}

All results for the stability samples tested are summarized in Table 4 , and they were within the acceptable criteria of \pm $15 \%$, indicating that DEZ, MDZ and 1-OH MDZ were stable under the four conditions described.

\section{Effects of DEX on Pharmacokinetic of DEZ}

The plasma concentration-time curve of DEZ in group A and group B is shown in Figure 3. DAS 2.0 program was used to calculate the main pharmacokinetic parameters of DEZ, including t1/2, Tmax, Cmax, CLz/F, Vz/F, AUC(0-t), AUC $(0-)$. Meanwhile, Table 5 shows the main pharmacokinetic parameters of DEZ.

Results show that the Cmax, $\operatorname{AUC}\left(0^{-}-\mathrm{t}\right)$ and $\operatorname{AUC}\left(0^{-}\right)$ of DEZ in group B were $32.48 \%, 64.44 \%$, and $65.86 \%$ higher than that in group A, respectively, and the $11 / 2$ was increased from $0.74 \mathrm{~h}$ to $2.27 \mathrm{hrs}$, The $\mathrm{Vz} / \mathrm{F}$ increased and $\mathrm{CLz} / \mathrm{F}$ was correspondingly decreased. DEX could affect the pharmacokinetics of DEZ in Beagles. It was suggested that DEX can inhibit the metabolism of DEZ and increase the exposure of DEZ in beagles.

\section{Effects of DEX on Pharmacokinetic of MDZ and I-OH MDZ}

The plasma concentration-time curve of $\mathrm{MDZ}$ and 1-OH MDZ in group A and group B were shown in Figures 4 and 5. DAS 2.0 program was used to calculate the main pharmacokinetic parameters of MDZ and 1-OH MDZ and the main pharmacokinetic parameters are showed in Table 5 too.

Results show that the Cmax, $\operatorname{AUC}\left(0^{-}-\mathrm{t}\right)$ and $\mathrm{AUC}\left(0^{-}\right)$ of MDZ in group B were $43.81 \%, 75.81 \%$ and $65.46 \%$ higher than that in group A, respectively, and the $t 1 / 2$ was increased from $1.18 \mathrm{~h}$ to $2.64 \mathrm{~h}$. The $\mathrm{Vz} / \mathrm{F}$ increased and $\mathrm{CLz} / \mathrm{F}$ was correspondingly decreased. DEX could affect the pharmacokinetics of MDZ in Beagles. It was suggested that DEX could inhibit the metabolism of MDZ and increase the exposure of MDZ in beagles. At the same time, the Cmax of 1-OH MDZ in group B was $29.81 \%$ lower than that in group $\mathrm{A}$, but the $\mathrm{t} 1 / 2$ was increased from $1.27 \mathrm{~h}$ to $2.34 \mathrm{~h}$. There was no significant change in AUC between the two groups.

\section{Discussion}

In this study, a novel UPLC-MS/MS method for simultaneous determination of the concentrations of DEX, DEZ and MDZ in Beagles plasma was developed. This method has high sensitivity, rapid detection time (3 min), improve detection standards and reliability, and met the requirements of pharmacokinetic guidelines. Acetonitrile was used for protein precipitation, which was simpler and reliable. Methanol, acetonitrile, and ultrapure water were 

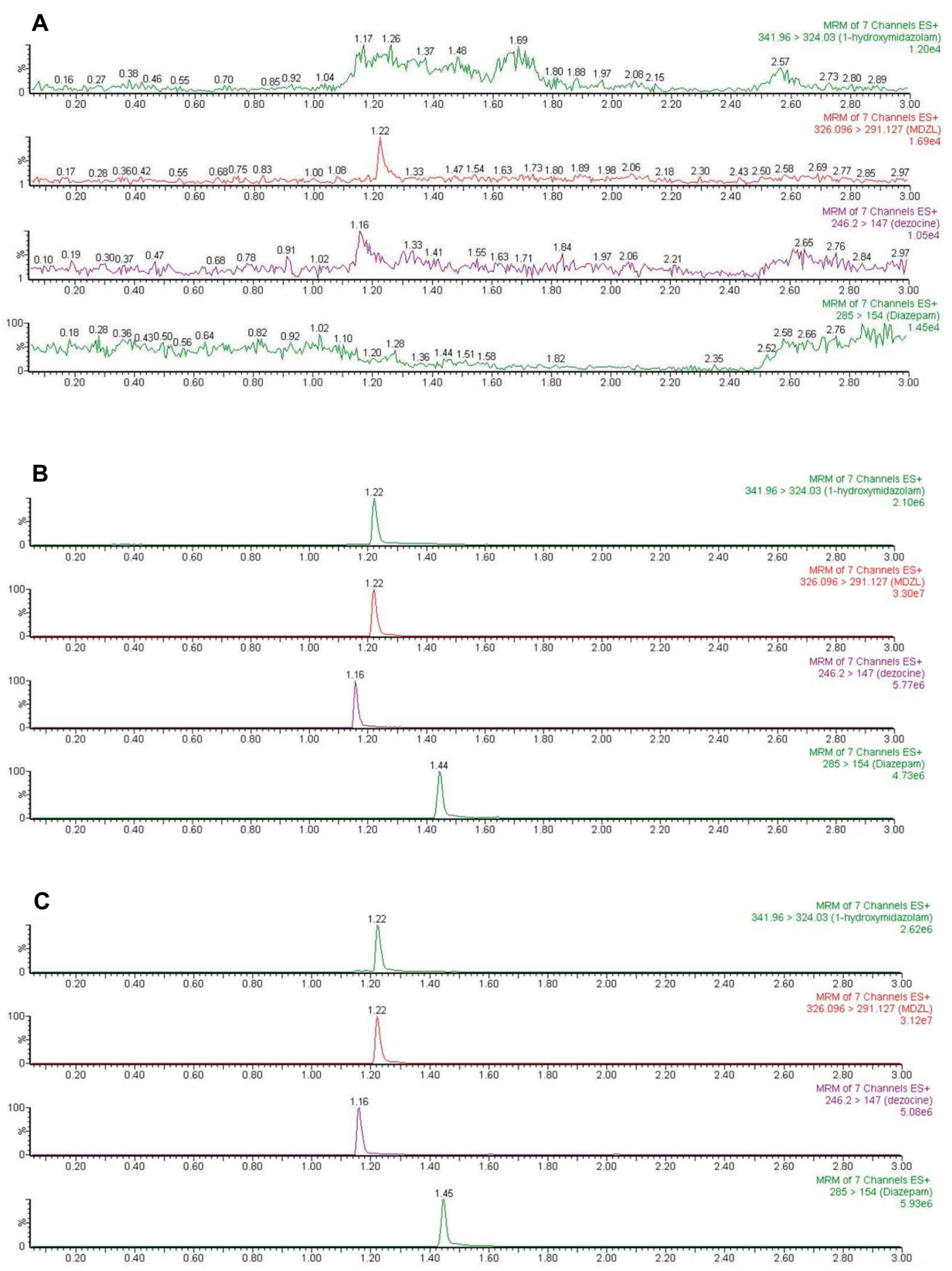

Figure 2 Representative chromatograms in positive ion mode. (A) A blank plasma sample; (B) A blank plasma sample spiked with DEZ, MDZ, I-OH MDZ and IS; (C) A beagle plasma sample $3.0 \mathrm{~h}$ after injection of DEZ and MDZ.

studied as solvents, respectively. The best resolution was obtained after methanol dissolution by UPLC-MS/MS analysis. In this study, Diazepam was used as the IS, because diazepam was stable and easy to obtain. It has a similar peak time with DEX, DEZ and MDZ, and the endogenous substances in the Beagles plasma samples did not affect its content determination. Compared with the research report by Cui et $\mathrm{al}^{20}$ our experimental subjects were Beagles, 
Table I Regression Equation, Linear Ranges, Correlation Coefficients and LLOQ of Three Analytes

\begin{tabular}{|l|l|l|l|l|}
\hline Analytes & Regression Equation & Linear Ranges $(\mathbf{n g} / \mathbf{m L})$ & $\mathbf{R}$ & LLOQ (ng/mL) \\
\hline DEZ & $y=0.0176 x+0.0042$ & $1-500$ & 0.9991 & 1.00 \\
MDZ & $y=0.1251 x+0.2339$ & $0.5-100$ & 0.9984 & 0.50 \\
I-OH MDZ & $y=0.0064 x+0.0200$ & $1-500$ & 0.9954 & 1.00 \\
\hline
\end{tabular}

Table 2 Precision and Accuracy of Three Analytes in Beagle Plasma $(n=6$, Mean \pm SD)

\begin{tabular}{|l|l|l|l|l|l|l|}
\hline \multirow{2}{*}{ Analytes } & Spiked (ng/mL) & \multicolumn{2}{l|}{ Intra-Day } & \multicolumn{2}{l|}{ Inter-Day } \\
\cline { 3 - 7 } & & RSD (\%) & RE (\%) & \multicolumn{2}{l|}{ RSD (\%) } & RE (\%) \\
\hline \multirow{2}{*}{ DEZ } & 1 & 3.59 & -1.57 & 9.58 & 7.66 \\
& 2.5 & 6.85 & 5.06 & 0.37 & 7.78 \\
& 50 & 7.66 & 0.93 & 6.79 & 0.43 \\
& 400 & 6.26 & -2.53 & 0.26 & 0.47 \\
\hline \multirow{2}{*}{ MDZ } & 0.5 & 6.14 & 2.58 & 7.36 & 4.19 \\
& 1 & 3.34 & -4.43 & 9.63 & -4.65 \\
& 10 & 6.24 & 1.32 & 3.86 & -1.64 \\
& 80 & 3.09 & 2.14 & 4.58 & 2.53 \\
\hline \multirow{2}{*}{ I-OH MDZ } & 1 & 5.90 & 4.73 & 10.09 & 3.65 \\
& 2.5 & 4.55 & -4.66 & 7.91 & -3.47 \\
& 50 & 7.81 & 7.06 & 2.56 & 8.22 & 4.93 \\
& 400 & & 6.43 & 5.04 \\
\hline
\end{tabular}

Table 3 The Recoveries and ME of the Three Analytes in Beagle Plasma ( $n=6$, Mean \pm SD)

\begin{tabular}{|l|l|l|l|l|l|}
\hline Analytes & Spiked (ng/mL) & Recoveries (\%) & RSD (\%) & ME (\%) & RSD (\%) \\
\hline \multirow{2}{*}{ DEZ } & 2.5 & $86.99 \pm 3.37$ & 3.87 & $106.74 \pm 5.73$ & 5.39 \\
& 50 & $81.32 \pm 5.54$ & 6.81 & $108.61 \pm 6.93$ & 6.38 \\
& 400 & $87.21 \pm 6.73$ & 7.72 & $103.64 \pm 6.02$ & 5.81 \\
\hline \multirow{2}{*}{ MDZ } & 1 & $78.65 \pm 4.43$ & 5.63 & $105.86 \pm 8.87$ & 6.30 \\
& 10 & $84.08 \pm 5.91$ & 6.97 & $97.59 \pm 6.30$ & 6.46 \\
& 80 & $85.24 \pm 3.66$ & 4.29 & $97.35 \pm 5.86$ & 6.02 \\
\hline \multirow{2}{*}{ I-OH MDZ } & 2.5 & $83.17 \pm 4.07$ & 4.89 & $99.02 \pm 7.22$ & 7.29 \\
& 50 & $85.39 \pm 5.49$ & 6.43 & $100.02 \pm 4.02$ & 4.02 \\
& 400 & $86.22 \pm 3.01$ & 3.49 & $104.83 \pm 6.82$ & 6.51 \\
\hline
\end{tabular}

whose metabolism was closer to humans. In addition, simpler plasma processing method was used in our study.

Drug-drug interactions (DDI) will occur when the pharmacokinetics (PKs) and pharmacodynamics (PDs) of a drug are altered by one or more drugs. ${ }^{21}$ DDI can significantly affect patient safety, so it is important to assess the risks of DDI. ${ }^{22}$ Pharmacokinetics (PKs) can occur at any stage of absorption, distribution, metabolism, and excretion, but primarily at the metabolic stage of the drug. ${ }^{23}$ A primary reason of DDIs is the change of the cytochrome P450
(CYP450) isozyme activity by inducing or inhibiting. ${ }^{24,25}$ The hepatic cytochrome P450 (CYP) enzyme system is the main site where drug metabolism and $86 \%$ drug interaction occur.

The DDIs that result in complicated outcomes, such as an increase of serious adverse reactions, lack of efficacy, or tolerability issues, may be clinically significant. The clinical relevance of many DDIs is easily underestimated, as detection is challenging and possible outcomes may vary widely. Therefore, it is critical to avoid DDIs where 
Table 4 The Stability of the Three Analytes in Beagle Plasma $(n=6$, Mean \pm SD)

\begin{tabular}{|c|c|c|c|c|c|c|c|c|c|}
\hline \multirow[t]{2}{*}{ Analytes } & \multirow[t]{2}{*}{ Spiked (ng/mL) } & \multicolumn{2}{|c|}{ Room Temperature, $12 \mathrm{~h}$} & \multicolumn{2}{|c|}{ Autosampler $4^{\circ} \mathrm{C}, 12 \mathrm{~h}$} & \multicolumn{2}{|c|}{ Three Freeze-Thaw } & \multicolumn{2}{|c|}{$-20^{\circ} \mathrm{C}, 4$ Weeks } \\
\hline & & RSD (\%) & RE (\%) & RSD (\%) & RE (\%) & RSD (\%) & RE (\%) & RSD (\%) & RE (\%) \\
\hline \multirow[t]{3}{*}{ DEZ } & 2.5 & 6.69 & 2.25 & 5.07 & 4.58 & $1 \mathrm{l} .42$ & 3.29 & $1 \mathrm{I} .74$ & 3.84 \\
\hline & 50 & 9.48 & -4.05 & 0.02 & -0.17 & 9.72 & 3.68 & 6.00 & -0.90 \\
\hline & 400 & 11.94 & 2.72 & 9.32 & 2.87 & 1.51 & 2.85 & 6.82 & 2.23 \\
\hline \multirow[t]{3}{*}{ MDZ } & 1 & 1.26 & -4.57 & 7.10 & 3.87 & 1.48 & -0.02 & 9.46 & 1.32 \\
\hline & 10 & 3.99 & 1.64 & 1.59 & -3.57 & 3.24 & -0.19 & 0.45 & 3.68 \\
\hline & 80 & 1.09 & -3.33 & 3.92 & -1.29 & 7.02 & 3.64 & 5.53 & 4.42 \\
\hline \multirow[t]{3}{*}{ I-OH MDZ } & 2.5 & 5.43 & 4.09 & 7.21 & 0.23 & 8.64 & -4.23 & 1.90 & -4.92 \\
\hline & 50 & 9.34 & -3.97 & 4.81 & 1.75 & 10.92 & 0.26 & 10.97 & 2.35 \\
\hline & 400 & 3.12 & 0.56 & 10.99 & 0.21 & 3.53 & -4.02 & 6.27 & -0.24 \\
\hline
\end{tabular}

possible through the selection of appropriate medications with the least potential for DDIs. ${ }^{3}$ At the same time, it is very important to identify the potential DDIs in order to reduce the risks of unexpected outcomes.

DEX is a highly selective $\alpha 2$-adrenoceptor agonist with sedative, anxiolytic, sympatholytic and analgosedative properties. ${ }^{1}$ DEX is metabolised by glucuronidation and CYP2A6 hydroxylation and subsequently excreted in urine almost exclusively as a metabolite. ${ }^{4,5}$ DEX is a strong inhibitor of cytochrome CYP450 enzyme. ${ }^{18}$ The combination of sufentanil and DEX can cause drug-drug interactions, which may promote the sedation and prolong the respiratory depression by increasing the exposure level of DEX brain tissue. In clinical application, attention should be paid to the possible drug-drug interactions or adverse reactions caused by the combination of these two drugs. ${ }^{18}$ DEX enhances the action of mepivacaine in reducing regional blood flow prolongs its tissue retention, and increases the local anesthetic action without affecting hemodynamics on local administration. ${ }^{26}$

In infants and toddlers, DEX reached its peak within $47 \mathrm{mins}$ after intranasal administration $1 \mathrm{mg} / \mathrm{kg}$ or $2 \mathrm{mg} / \mathrm{kg}$, with an overall bioavailability of $84 \%$ and the Cmax were 182 $\mathrm{pg} / \mathrm{mL}$ and $324 \mathrm{pg} / \mathrm{mL} .{ }^{27} \mathrm{In} \mathrm{SD}$ rats, the $\mathrm{t} 1 / 2$ after intravenous injection of DEX (20 $\mu \mathrm{g} / \mathrm{kg})$, DEZ (80 $\mu \mathrm{g} / \mathrm{kg})$, and MID (400 $\mu \mathrm{g} / \mathrm{kg}$ ) were $141.25 \mathrm{~min}, 225.39 \mathrm{~min}$, and $80.51 \mathrm{~min}$, respectively. The Cmax values were $23.24 \mathrm{ng} / \mathrm{mL}, 22.37 \mathrm{ng} / \mathrm{mL}$ and $645.68 \mathrm{ng} / \mathrm{mL}$, respectively. ${ }^{20}$

The results of this study show that the Cmax, $\operatorname{AUC}(0-\mathrm{t})$ and $\mathrm{AUC}\left({ }^{-}\right)$of DEZ in group B were $32.48 \%, 64.44 \%$, and

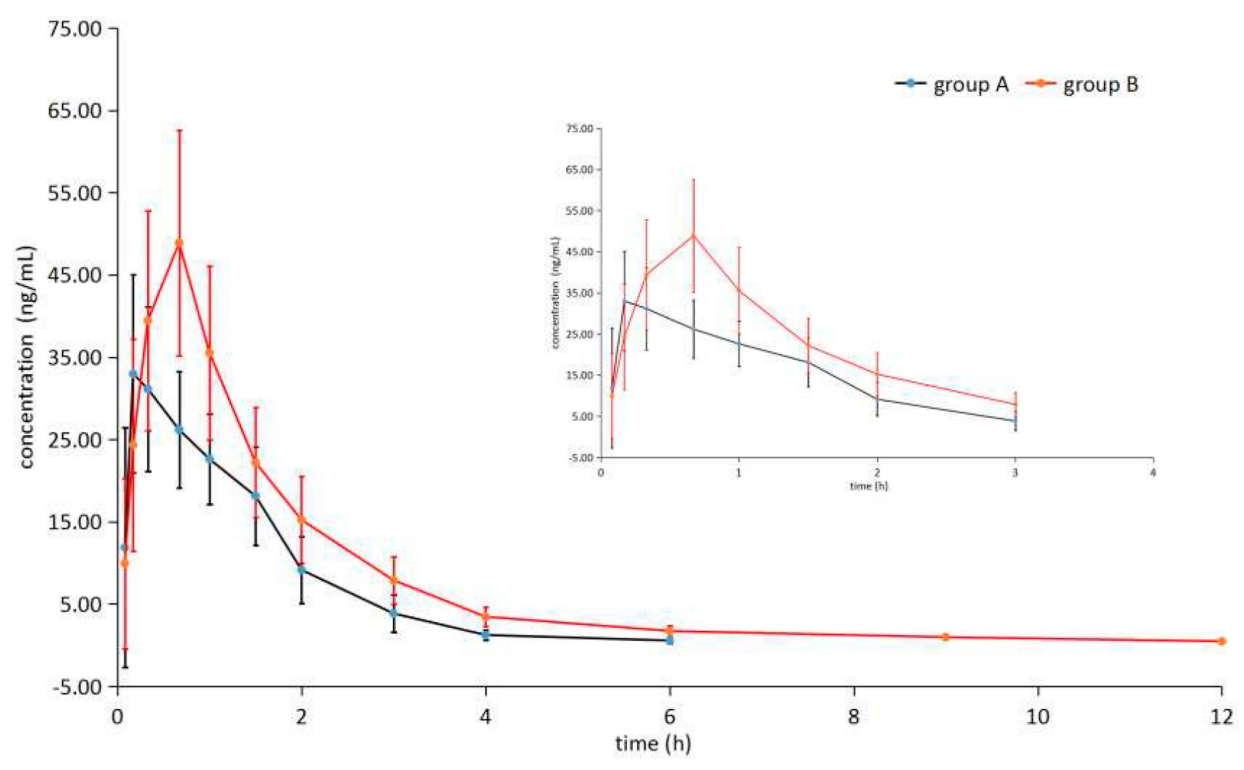

Figure 3 The mean concentration-time curve of DEZ in group A and group B. 
Table 5 Pharmacokinetic Parameters of DEZ, MDZ and I-OH MDZ in Group A and Group B ( $n=6$, Mean \pm SD)

\begin{tabular}{|c|c|c|c|c|c|c|}
\hline \multirow[t]{2}{*}{ Parameters } & \multicolumn{2}{|l|}{ DEZ } & \multicolumn{2}{|l|}{ MDZ } & \multicolumn{2}{|l|}{ I-OH MDZ } \\
\hline & Group A & Group B & Group A & Group B & Group A & Group B \\
\hline $\mathrm{tl} / 2(\mathrm{~h})$ & $0.74 \pm 0.25$ & $2.27 \pm 0.491$ & $1.18 \pm 0.41$ & $2.64 \pm 0.91$ & $1.27 \pm 1.02$ & $2.34 \pm 0.74$ \\
\hline $\operatorname{Tmax}(h)$ & $0.25 \pm 0.09$ & $0.61 \pm 0.14$ & $0.22 \pm 0.08$ & $0.34 \pm 0.26$ & $0.30 \pm 0.07$ & $0.53 \pm 0.22$ \\
\hline $\mathrm{Cmax}(\mathrm{ng} / \mathrm{mL})$ & $38.02 \pm 11.32$ & $50.37 \pm 11.96$ & $49.72 \pm 21.10$ & $71.24 \pm 17.12$ & $95.7 I \pm 32.37$ & $67.18 \pm 22.22$ \\
\hline CLz/F (L/h/kg) & $6.57 \pm 1.95$ & $3.92 \pm 1.07$ & $3.47 \pm 1.46$ & $1.75 \pm 0.54$ & $1.37 \pm 0.40$ & $1.52 \pm 0.39$ \\
\hline $\mathrm{Vz} / \mathrm{F}(\mathrm{L} / \mathrm{kg})$ & $6.82 \pm 2.26$ & $12.09 \pm 3.98$ & $6.18 \pm 4.46$ & $7.08 \pm 4.34$ & $2.53 \pm 1.91$ & $5.04 \pm 1.75$ \\
\hline$A \cup C(0-t)$ & $53.23 \pm$ & $87.53 \pm$ & $52.33 \pm$ & $92.00 \pm$ & $118.91 \pm$ & $113.80 \pm$ \\
\hline$(\mathrm{ng} \cdot \mathrm{h} / \mathrm{mL})^{*}$ & 14.15 & 22.52 & 29.04 & 38.29 & 45.03 & 54.55 \\
\hline $\operatorname{A\cup C}_{(0-\infty)}$ & $53.80 \pm$ & $89.23 \pm$ & $57.26 \pm$ & $94.74 \pm$ & $120.64 \pm$ & $116.27 \pm$ \\
\hline (ng h/mL) & 14.57 & 22.60 & 34.25 & 37.23 & 44.52 & 55.33 \\
\hline
\end{tabular}

Notes: AUC(0-t) in Group A was AUC of 0-6 h, AUC(0-t) in Group B was AUC of 0-12 hrs.

$65.86 \%$ higher than that in group A, respectively, and the $\mathrm{t} 1 / 2$ was increased from $0.74 \mathrm{~h}$ to $2.27 \mathrm{~h}$. The $\mathrm{Vz} / \mathrm{F}$ increased and $\mathrm{CLz} / \mathrm{F}$ was correspondingly decreased. It was suggested that DEX can inhibit the metabolism of DEZ and increase the exposure of DEZ in beagles. From the pharmacokinetic point of view, the analgesic effect was enhanced with the increase of serum concentration of DEZ.

The results of this study show that the Cmax, $\operatorname{AUC}(0-\mathrm{t})$ and $\mathrm{AUC}\left(0^{-}\right)$of $\mathrm{MDZ}$ in group B were $\%, 43.28 \%, 75.81 \%$ and $65.46 \%$ higher than that in group A, respectively, and the $\mathrm{t} 1 / 2$ was increased from $1.18 \mathrm{~h}$ to $2.64 \mathrm{~h}$. The $\mathrm{CLz} / \mathrm{F}$ was decreased, and the $\mathrm{Vz} / \mathrm{F}$ was increased, respectively. The Cmax of 1-OH MDZ in group B was $29.81 \%$ lower than that in group $\mathrm{A}$, but the $\mathrm{t} 1 / 2$ was increased from $1.27 \mathrm{~h}$ to 2.34 h. There was no significant change in AUC between the two groups. The $\mathrm{CLz} / \mathrm{F}$ and the $\mathrm{Vz} / \mathrm{F}$ were increased. It was suggested that DEX can inhibit the metabolism of MDZ and increase the exposure of MDZ in beagles. From the pharmacokinetic point of view, the analgesic effect was enhanced with the increase of serum concentration of MDZ.

The last time point of blood collection in this study was $12 \mathrm{hrs}$ after administration. It was found that the concentration of the three analytes in group A decreased to the LLOQ at $6 \mathrm{hrs}$, while that in group B decreased to the LLOQ at 12 hrs. Therefore, different time was chosen in the calculation of pharmacokinetic parameters.

Although there are some differences in metabolism between different species, the results of animal experiments could provide a reference for clinical medication. ${ }^{28}$ Therefore, clinically, the change of therapeutic effect and

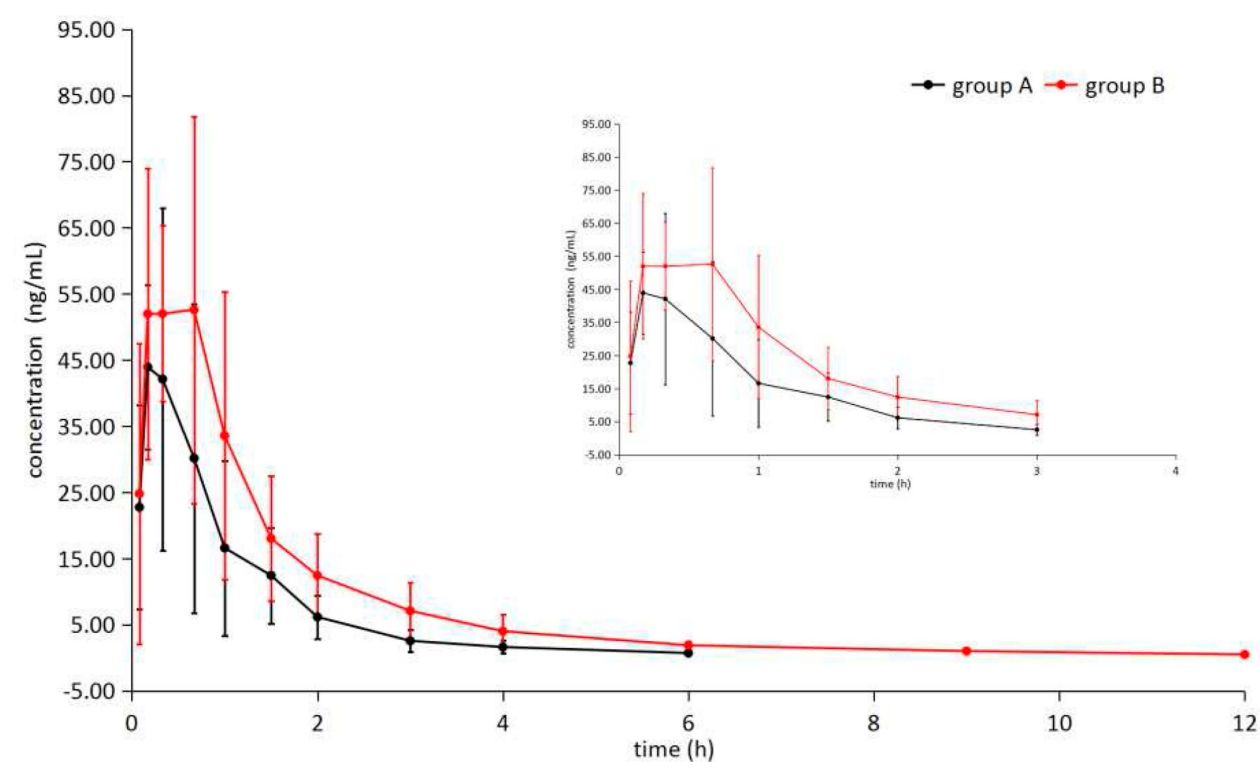

Figure 4 The mean concentration-time curve of $M D Z$ in group $A$ and group $B$. 


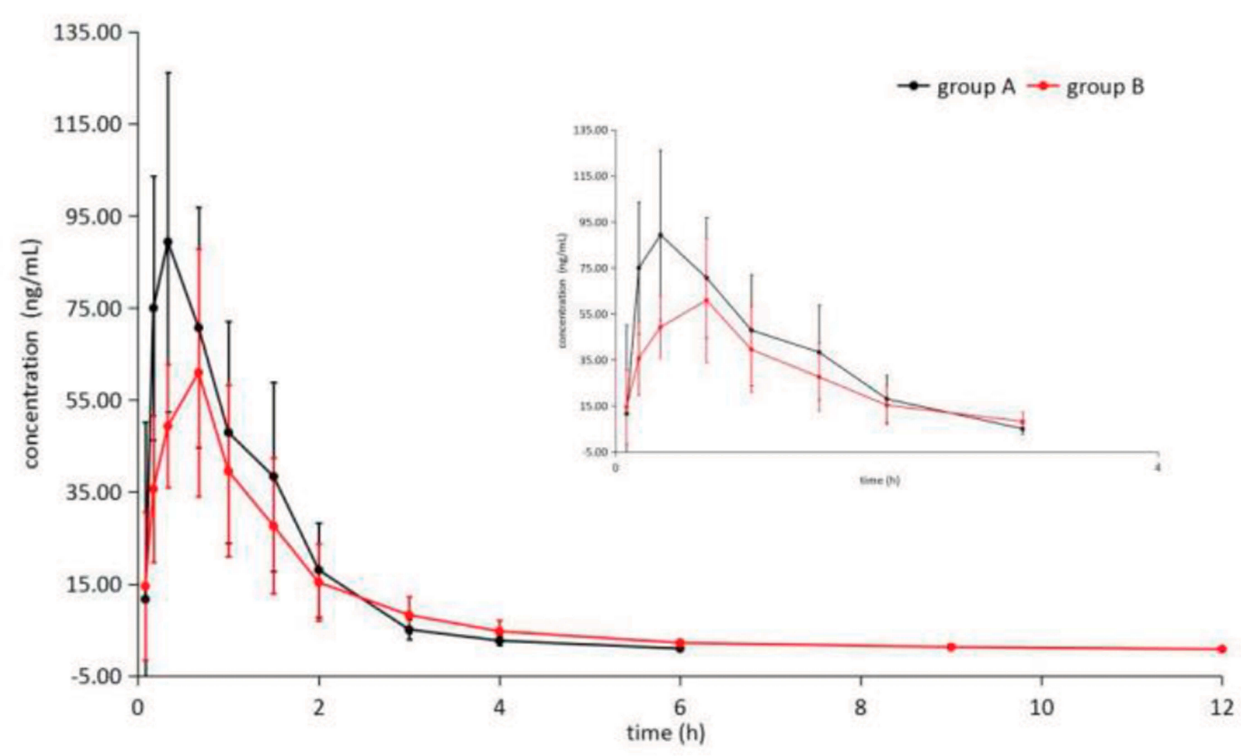

Figure 5 The mean concentration-time curve of $\mathrm{I}-\mathrm{OH}$ MDZ in group A and group $\mathrm{B}$.

the occurrence of adverse reactions caused by drug-drug interaction should be paid attention to when DEX was combined with DEZ and MDZ.

\section{Conclusion}

The developed UPLC-MS/MS method for simultaneous determination of DEZ, MDZ and its metabolite 1-OH MDZ in beagles plasma was accurate, reproducible, specific. DEX could inhibit the metabolism of DEZ and MDZ and increase the exposure of DEZ and MDZ in beagles. Therefore, the change of therapeutic effect and the occurrence of adverse reactions caused by drug-drug interaction should be paid attention to when the drugs were used in combination.

\section{Data Sharing Statement}

The data used to support the findings of this study are available from the corresponding authors upon request. The corresponding authors: Xiang-jun Qiu (lyxiangjun@126.com) and Wei Zhang (zhangw571012@126. com).

\section{Acknowledgments}

This study was supported by grants from Nanyang City Central Hospital. We appreciate the contribution of the members participating in this study. We also would like to thank the Wenzhou Medical University for support with the equipment for this work.

\section{Author Contributions}

All authors contributed to data analysis, drafting or revising the article, gave final approval of the version to be published, and agree to be accountable for all aspects of the work.

\section{Disclosure}

The authors report no conflicts of interest in this work.

\section{References}

1. Lin Q, Xie S, Qiu X, Chen J, Xu RA. Drug-drug interaction study of imatinib and voriconazole in vitro and in vivo. Infect Drug Resist. 2019;12:1021-1027. doi:10.2147/IDR.S199526

2. Roberts AG, Gibbs ME. Mechanisms and the clinical relevance of complex drug-drug interactions. Clin Pharmacol. 2018;10:123-134. doi:10.2147/CPAA.S146115

3. Low Y, Setia S, Lima G. Drug-drug interactions involving antidepressants: focus on desvenlafaxine. Neuropsychiatr Dis Treat. 2018;14:567-580. doi:10.2147/NDT.S157708

4. Keating GM. Dexmedetomidine: a review of its use for sedation in the intensive care setting. Drugs. 2015;75(10):1119-1130. doi:10.1007/ s40265-015-0419-5

5. Wang H, Tian Q, Quan P, Liu C, Fang L. Probing the role of ion-pair strategy in controlling dexmedetomidine penetrate through drug-inadhesive patch: mechanistic insights based on release and percutaneous absorption process. AAPS PharmSciTech. 2019;21(1):4. doi:10.1208/s12249-019-1539-0

6. Weerink MAS, Struys M, Hannivoort LN, Barends CRM, Absalom AR, Colin P. Clinical pharmacokinetics and pharmacodynamics of dexmedetomidine. Clin Pharmacokinet. 2017;56(8):893-913. doi:10.1007/s40262-017-0507-7

7. Wang Y, Fang X, Liu C, et al. Impact of intraoperative infusion and postoperative PCIA of dexmedetomidine on early breastfeeding after elective cesarean section: a randomized double-blind controlled trial. Drug Des Devel Ther. 2020;14:1083-1093. doi:10.2147/DDDT. S241153 
8. van Dijkman SC, De Cock P, Smets $\mathrm{K}$, et al. Dose rationale and pharmacokinetics of dexmedetomidine in mechanically ventilated new-borns: impact of design optimisation. Eur J Clin Pharmacol. 2019;75(10):1393-1404. doi:10.1007/s00228-019-02708-y

9. Kohli U, Pandharipande P, Muszkat M, et al. CYP2A6 genetic variation and dexmedetomidine disposition. Eur J Clin Pharmacol. 2012;68(6):937-942. doi:10.1007/s00228-011-1208-z

10. Wang YH, Chai JR, Xu XJ, et al. Pharmacological characterization of dezocine, a potent analgesic acting as a kappa partial agonist and $\mathrm{Mu}$ partial agonist. Sci Rep. 2018;8(1):14087. doi:10.1038/s41598-01832568-y

11. Liu R, Huang XP, Yeliseev A, Xi J, Roth BL. Novel molecular targets of dezocine and their clinical implications. Anesthesiology. 2014;120 (3):714-723. doi:10.1097/ALN.0000000000000076

12. Zhou C, Yang Y, Zhu Y, Ruan L. Effects of dezocine on prevention of propofol injection pain: a meta-analysis. J Pain Res. 2017;10:13691375. doi:10.2147/JPR.S128889

13. Steiner C, Steurer MP, Mueller D, Zueger M, Dullenkopf A. Midazolam plasma concentration after anesthesia premedication in clinical routine - an observational study: midazolam plasma concentration after anesthesia premedication. BMC Anesthesiol. 2016;16 (1):105. doi:10.1186/s12871-016-0262-6

14. Kawai M, Kurata S, Sanuki T, et al. The effect of midazolam administration for the prevention of emergence agitation in pediatric patients with extreme fear and non-cooperation undergoing dental treatment under sevoflurane anesthesia, a double-blind, randomized study. Drug Des Devel Ther. 2019;13:1729-1737. doi:10.2147/DDDT.S198123

15. Neuman G, Swed Tobia R, Koren L, Leiba R, Shavit I. Single dose oral midazolam for minor emergency department procedures in children: a retrospective cohort study. J Pain Res. 2018;11:319-324. doi:10.2147/JPR.S156080

16. Blumer JL. Clinical pharmacology of midazolam in infants and children. Clin Pharmacokinet. 1998;35(1):37-47. doi:10.2165 00003088-199835010-00003

17. Wu ML, Lin YP, Wei YL, et al. Calycosin influences the metabolism of five probe drugs in rats. Drug Des Devel Ther. 2020;14:429-434. doi:10.2147/DDDT.S236221

18. Chang X, YU CC, Hua L, et al. Pharmacokinetics and sedation of sufentanil and dexmedetomidine combination in rats. Chin $J$ Pharmacol Toxicol. 2019;33(1):63-69.
19. US Food and Drug Administration. Guidance for Indus-Try: Bioanalytical Method Validation. Rockville, MD, USA: US Department of Health and Human Services, US FDA, Center for Drug Evaluation and Research; 2018.

20. Cui W, Liu Q, Xiong S, Qiao L. LC-MS/MS method for simultaneous quantification of dexmedetomidine, dezocine, and midazolam in rat plasma and its application to their pharmacokinetic study. J Anal Methods Chem. 2018;2018:3184759. doi:10.1155/2018/3184759

21. Varma MV, Pang KS, Isoherranen N, Zhao P. Dealing with the complex drug-drug interactions: towards mechanistic models. Biopharm Drug Dispos. 2015;36(2):71-92. doi:10.1002/bdd.1934

22. Min JS, Bae SK. Prediction of drug-drug interaction potential using physiologically based pharmacokinetic modeling. Arch Pharm Res. 2017;40(12):1356-1379. doi:10.1007/s12272-017-0976-0

23. Di Gion P, Kanefendt F, Lindauer A, et al. Clinical pharmacokinetics of tyrosine kinase inhibitors: focus on pyrimidines, pyridines and pyrroles. Clin Pharmacokinet. 2011;50(9):551-603. doi:10.2165/ 11593320-000000000-00000

24. Gu H, Dutreix C, Rebello S, et al. Simultaneous physiologically based pharmacokinetic (PBPK) modeling of parent and active metabolites to investigate complex CYP3A4 drug-drug interaction potential: a case example of midostaurin. Drug Metab Dispos. 2018;46 (2):109-121. doi:10.1124/dmd.117.078006

25. Luo M, Dai M, Lin H, et al. Species-related exposure of Phase II metabolite gemfibrozil 1- O-beta-glucuronide between human and mice: a net induction of mouse P450 activity was revealed. Biopharm Drug Dispos. 2017;38(9):535-542. doi:10.1002/bdd.2105

26. Yasuda Y, Hashimoto S, Sunada K. Impact of dexmedetomidine on the tissue distribution, anesthetic action, and hemodynamic effects of mepivacaine. Odontology. 2019;107(1):29-36. doi:10.1007/s10266018-0368-z

27. Miller JW, Balyan R, Dong M, et al. Does intranasal dexmedetomidine provide adequate plasma concentrations for sedation in children: a pharmacokinetic study. Br J Anaesth. 2018;120(5):1056-1065. doi:10.1016/j.bja.2018.01.035

28. Shen X, Chen F, Wang F, Huang P, Luo W. The effect of grapefruit juice on the pharmacokinetics of tadalafil in rats. Biomed Res Int. 2020;2020:1631735. doi:10.1155/2020/1631735

\section{Publish your work in this journal}

Drug Design, Development and Therapy is an international, peerreviewed open-access journal that spans the spectrum of drug design and development through to clinical applications. Clinical outcomes, patient safety, and programs for the development and effective, safe, and sustained use of medicines are a feature of the journal, which has also been accepted for indexing on PubMed Central. The manuscript management system is completely online and includes a very quick and fair peer-review system, which is all easy to use. Visit http://www. dovepress.com/testimonials.php to read real quotes from published authors. 